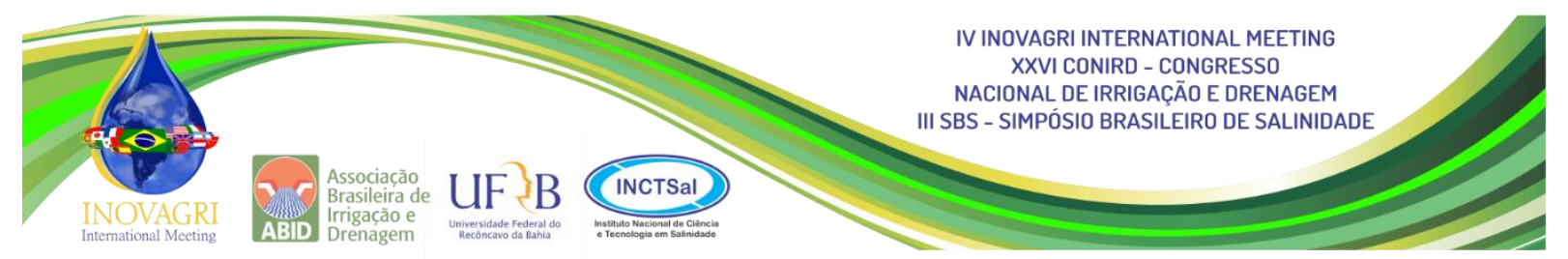

\title{
PRODUÇÃO DO MELÃO “AMARELO” SUBMETIDO A DISTINTOS SISTEMAS DE IRRIGAÇÃO E MULCHING EM PETROLINA-PE
}

\author{
W. de B. Pereira ${ }^{1}$, J. S. C. de Sousa ${ }^{2}$, M. E. P. dos $\operatorname{Santos}^{3}$, W. L. Simões ${ }^{4}$
}

RESUMO: O presente estudo foi realizado no Campus Petrolina Zona Rural do IF Sertão-PE, em Petrolina-PE, com o objetivo de avaliar a produção de híbridos de melão amarelo em dois sistemas de irrigação por gotejamento e três coberturas de solo no Vale do Submédio São Francisco. O delineamento experimental foi em blocos casualizados, esquema de parcelas subsubdivididas, 2 × 2 × 3 × 6, onde: 2 - híbridos de melão (Gladial e 10/00), 2 - sistemas de irrigação ( 1 fileira de gotejadores por fileira de plantas e 2 fileiras de gotejadores por fileira de planta), 3 - coberturas de solo (cobertura com mulching preto; cobertura com mulching cinza e sem cobertura de solo) e 6 - blocos (repetições). Observou-se que a produtividade diferiu-se estatisticamente para nível de efeito à $(\mathrm{P}<.01)$, em que a cobertura de solo com mulching cinza apresentou maior produtividade mesmo não diferindo estatisticamente do mulching preto, apresentando os melhores resultados em relação ao sem cobertura de solo. Os valores de produtividade em $\mathrm{kg} \mathrm{ha}^{-1}$ foram de 41.805,55; 36.597,22 e 27.500,00 respectivamente.

PALAVRAS-CHAVE: Cucumis Melo L., manejo de irrigação, solo coberto

\section{PRODUCTION OF "YELLOW" MELON SUBMITTED TO DIFFERENT IRRIGATION AND MULCHING SYSTEMS IN PETROLINA-PE}

\begin{abstract}
The present study was carried out at the Petrolina Campus of the Sertão-PE Rural Area, in Petrolina-PE, with the objective of evaluating the production of yellow melon hybrids in two drip irrigation systems and three soil coverages In the Valley of the Submédio San Francisco. The experimental design was a randomized block design, subdivided plots scheme, $2 \times 2 \times 3 \times 6$, where: 2 - hybrids of melon (gladial and 10/00), 2 - irrigation systems (1 row of drippers per row of plants And 2 rows of drippers per plant row), 3 - soil cover (black mulching cover, gray mulch cover and soil cover) and 6 - blocks (replicates). It was observed that

\footnotetext{
${ }^{1}$ Discente de graduação em agronomia, IF-Sertão CPZR, Petrolina - Pernambuco. Email: wiltem1993if@gmail.com.

${ }^{2}$ Doutor, Discente de graduação em agronomia, IF-Sertão CPZR, Petrolina - Pernambuco. Email: sebastiao.costa@ifsertao-pe.edu.br.

${ }^{3}$ Discente do técnico em agropecuária, IF-Sertão CPZR, Petrolina - Pernambuco. Email: mariaerica.3015@gmail.com.

${ }^{4}$ D.Sc. em Engenharia Agronômica, Pesquisador da Embrapa Semiárido, Petrolina - Pernambuco. Email: welson.simoes@embrapa.br.
} 
productivity differed statistically to effect level a $(\mathrm{P}<.01)$, in which the mulching gray soil showed higher productivity even though it did not differ statistically from the black mulching, presenting the best results in relation to the no Ground cover. The productivity values in $\mathrm{kg} \mathrm{ha}$

${ }^{1}$ were $41805.55 ; 36597.22$ and 27500.00 respectively.

KEY WORDS: Cucumis Melo L., irrigation management, soil covered

\section{INTRODUÇÃO}

O Submédio do Vale do São Francisco se destacar como polo da frutihorticultura nacional, nesta região semiárida do país, as elevadas produtividades e qualidade, é devido, sobretudo, as favoráveis condições climatológicas (em termos de luminosidade) e edáficas da região para estes cultivos (Lemos et al., 2012; EMBRAPA, 2016).

O meloeiro é uma olerícola pertencente à família das cucurbitáceas, originária da África e Ásia. E no nordeste o estado do Pernambuco é o quarto maior produtor e exportador de melão (Cucumis melo L.) do Brasil, (Crisóstomo, 2002).

Segundo Lima (2015) no Brasil as principais variedades de melão exploradas são do tipo Pele de Sapo, Amarelo, Cataloupe e Gália. Sendo que nos últimos anos os híbridos vêm sendo preferidos pela sua maior resistência a pragas, maiores e melhores produtividades, além de uniformidade dos melões. Só não existem relatos conclusivos de quanto se produz a mais com os híbridos.

No entanto não só a escolha da variedade defini o sucesso no cultivo de melão, mas está atrelado diretamente ao manejo praticado, ou seja, a irrigação, a adubação e os tratos culturais (condução da cultura, controle de pragas e doenças, cobertura do solo, etc.) (Batista et al., 2009).

Abreu et al. (1978) comentam ainda o trabalho de Shmueli \& Goldberg (1971), que investigaram o cultivo de melão sob irrigação por sulco, aspersão e gotejamento. No trabalho foi observando um desenvolvimento vegetativo mais rápido e uma maior produção com o gotejamento e nenhuma diferença significativa da cultura para os sistemas de irrigação por sulcos e aspersão.

Portanto, priorizam-se irrigações por gotejamento na cultura do meloeiro; a única dúvida é com relação à percentagem de solo molhado necessária para a cultura. O espaçamento praticado com o cultivo $(0,30$ a $0,50 \mathrm{~m}$ entre plantas e 2,00 m entre fileiras de plantas, Cavalcanti et al., 2008) e o uso de uma única fileira de gotejadores por fileira de plantas (diâmetro do bulbo molhado entorno de 0,50 m, Mantovani et al., 2009) geram percentagens de 
solo molhado menores ao recomendado pela literatura para regiões áridas e semiáridas, que é de 33\% (Vermeiren \& Jobling, 1997; Gomes, 1999; Bernardo et al., 2006).

Outra estratégia que vem gerando aumentos de produtividade e qualidade em melões é a cobertura do solo. Cultivos de melão manejados com mulching apresentou produtividades de até 39\% superior em comparação aos cultivos sem mulching (Medeiros et al., 2006). A cor do mulching utilizado também é um fator que influência a produção do melão. Gondim et al. (2009) ao avaliarem índices de crescimento, fisiológicos e de produção, em híbridos de meloeiros Cantaloupe submetidos a cultivo em solo descoberto e coberto com filmes de polietileno de diferentes cores, em Mossoró/RN, constataram melhores respostas de qualidades dos melões para os tratamentos com cobertura do solo com plásticos de cor marrom.

Assim o trabalho teve o objetivo de avaliar a produção e a qualidade de híbridos de melão amarelo em dois sistemas de irrigação por gotejamento e três coberturas de solo.

\section{MATERIAL E MÉTODOS}

O presente estudo foi conduzido no Campus Petrolina Zona Rural do IFSERTÃO-PE, em Petrolina - PE (latitude; $9^{\circ} 20^{\prime} 14.14$ ' ' longitude $40^{\circ} 42^{\prime} 01.27^{\prime}$ ' W; altitude de 418 metros), que de acordo com a classificação climática de Köppen apresenta clima BSwh', isto é, semiárido muito quente e com quadra chuvosa no verão atrasando-se para o outono (Azevedo et al., 2003).

O cultivo foi irrigado por gotejamento, com sistema de cobertura do solo tipo mulching e as cultivares utilizadas foram o híbrido Gladial e o híbrido 10/00, cultivada com espaçamento de 2,00 m entre fileiras x $0,30 \mathrm{~m}$ entre plantas.

O delineamento experimental adotado consistiu em blocos casualizados, esquema de parcelas subsubdivididas, 2 × 2 × 3 × 6, onde: 2 - híbridos de melão (parcelas: H1 - melão gladial e, H2 - melão 10/00), 2 - sistemas de irrigação (subparcelas: S1 - 1 fileira de gotejadores por fileira de plantas e, S2 - 2 fileiras de gotejadores por fileira de planta), 3 - coberturas de solo (subsubparcelas: C1 - cobertura com mulching preto; $\mathrm{C} 2$ - cobertura com mulching cinza e, C3 - sem cobertura de solo) e 6 - blocos (repetições). Na Figura 1 encontra-se ilustrada a o esquema de instalação do experimento. 


\begin{tabular}{|l|l|l|}
\multicolumn{3}{c}{ Bloco 1} \\
\hline H2S2C1 & H2S2C2 & H2S2C3 \\
\hline H2S1C1 & H2S1C3 & H2S1C2 \\
\hline H1S2C3 & H1S2C1 & H1S2C2 \\
\hline H1S1C2 & H1S1C1 & H1S1C3 \\
\hline \multicolumn{3}{|c|}{ Bloco 4 } \\
\hline H1S1C1 & H1S1C2 & H1S1C3 \\
\hline H1S2C3 & H1S2C1 & H1S2C2 \\
\hline H2S2C3 & H2S2C1 & H2S2C2 \\
\hline H2S1C1 & H2S1C3 & H2S1C2 \\
\hline
\end{tabular}

\begin{tabular}{|l|l|l|} 
Bloco 2 \\
\hline H1S1C1 & H1S1C3 & H1S1C2 \\
\hline H1S2C3 & H1S2C2 & H1S2C1 \\
\hline H2S2C2 & H2S2C1 & H2S2C3 \\
\hline H2S1C3 & H2S1C2 & H2S1C1 \\
\hline \multicolumn{3}{|c|}{ Bloco 5 } \\
\hline H1S2C1 & H1S2C3 & H1S2C2 \\
\hline H1S1C2 & HIS1C1 & H1S1C3 \\
\hline H2S1C1 & H2S1C3 & H2S1C2 \\
\hline H2S2C3 & H2S2C2 & H2S2C1 \\
\hline
\end{tabular}

\begin{tabular}{|l|l|l|} 
Bloco 3 \\
\hline H2S1C3 & H2S1C1 & H2S1C2 \\
\hline H2S2C3 & H2S2C2 & H2S2C1 \\
\hline H1S1C2 & H1S1C1 & H1S1C3 \\
\hline H1S2C2 & H1S2C3 & $H 1 S 2 C 1$ \\
\hline \multicolumn{3}{|c|}{ Bloco 6 } \\
\hline H2S2C3 & H2S2C1 & H2S2C2 \\
\hline H2S1C2 & H2S1C3 & H2S1C1 \\
\hline H1S1C3 & H1S1C1 & H1S1C2 \\
\hline H1S2C2 & H1S2C3 & $H 1 S 2 C 1$ \\
\hline
\end{tabular}

Figura 1. Disposição dos tratamentos na área experimental. Onde: H1 - melão gladial; H2 - melão 10/00; S1 - uma fileira de gotejadores por fileira de plantas; S2 - duas fileiras de gotejadores por fileira de plantas; $\mathrm{C} 1$ - solo coberto com mulching preto; $\mathrm{C} 2$ - solo coberto com mulching cinza; $\mathrm{C} 3$ - solo sem cobertura.

O preparo do solo aconteceu inicialmente com aração e gradagem. Após esse preparo foram levantados canteiros de 0,30 $\mathrm{m}$ de largura, com altura média de 0,25 $\mathrm{m}$ e comprimento de 25,00 m para receber o sistema de irrigação, a cobertura do solo (com e sem mulching) e as mudas.

As mudas foram preparadas em bandejas de isopor com o semeio de uma semente por célula. As mudas permaneceram em sistema hidropônico durante duas semanas para o transplantio.

Antes do transplantio realizou-se a coleta de solo para análise físico-químico (permitindo o manejo da adubação, além da determinação da curva de retenção de água no solo). A adubação foi de acordo recomendação de Cavalcanti et al. (2008) e aplicação ocorreu via sistema de irrigação.

Realizou-se avaliação do sistema de irrigação de (uniformidade de distribuição de água e testes de pressão) antes e depois do ciclo produtivo a partir das metodologias de Bernardo et al. (2009) e Mantovani et al. (2009).

Foram instalados dois tensiômetros de punção (a 0,20 e 0,40 m de profundidade) em três parcelas experimentais, sendo 36 tensiômetros a 0,20 m de profundidade, 36 tensiômetros a 0,40 $\mathrm{m}$ de profundidade, para monitoramento da umidade do solo. Está variável foram computados a partir de informações tensiométricas, aferidas três vezes por semana com uso de tensímetro digital.

Os dados de produção do meloeiro foi determinado no momento da colheita, em que mesmos foram colhidos por subsubparcelas e pesados, registrados seus respectivos valores.

O manejo da irrigação realizou-se a partir do balanço hídrico sequencial da cultura, com dados meteorológicos provenientes de uma estação meteorológica automática, modelo Vantage 
Pro2, marca Devis do Campus Petrolina Zona Rural (instalada a cerca de $900 \mathrm{~m}$ da área experimental), valores de coeficiente de cultura (kc) apresentados pela FAO (Allen et al., 1998) e metodologia de Vermeiren \& Jobling (1997).

Os dados foram submetidos ao teste de médias. Para os fatores quantitativos foi utilizado o teste de Tukey a 5\% de probabilidade, a partir do programa computacional Assistat versão 7.7 Beta (Silva, 2014).

\section{RESULTADOS E DISCUSSÃO}

$\mathrm{Na}$ Tabela 1, estão apresentados dados produtivos dos melões híbridos Gladial e 10/00 em dois sistemas de irrigação por gotejamento (com uma e duas fileiras de gotejadores por fileira de planta) e três coberturas de solo (com mulching preto, mulching cinza e sem mulching) cultivado em Petrolina/PE.

Tabela 1. Resultados gerais para produção de híbridos de melão amarelo submetidos a diferentes sistemas de irrigação e coberturas do solo. Petrolina, IFSERTÃO-PE, 2016.

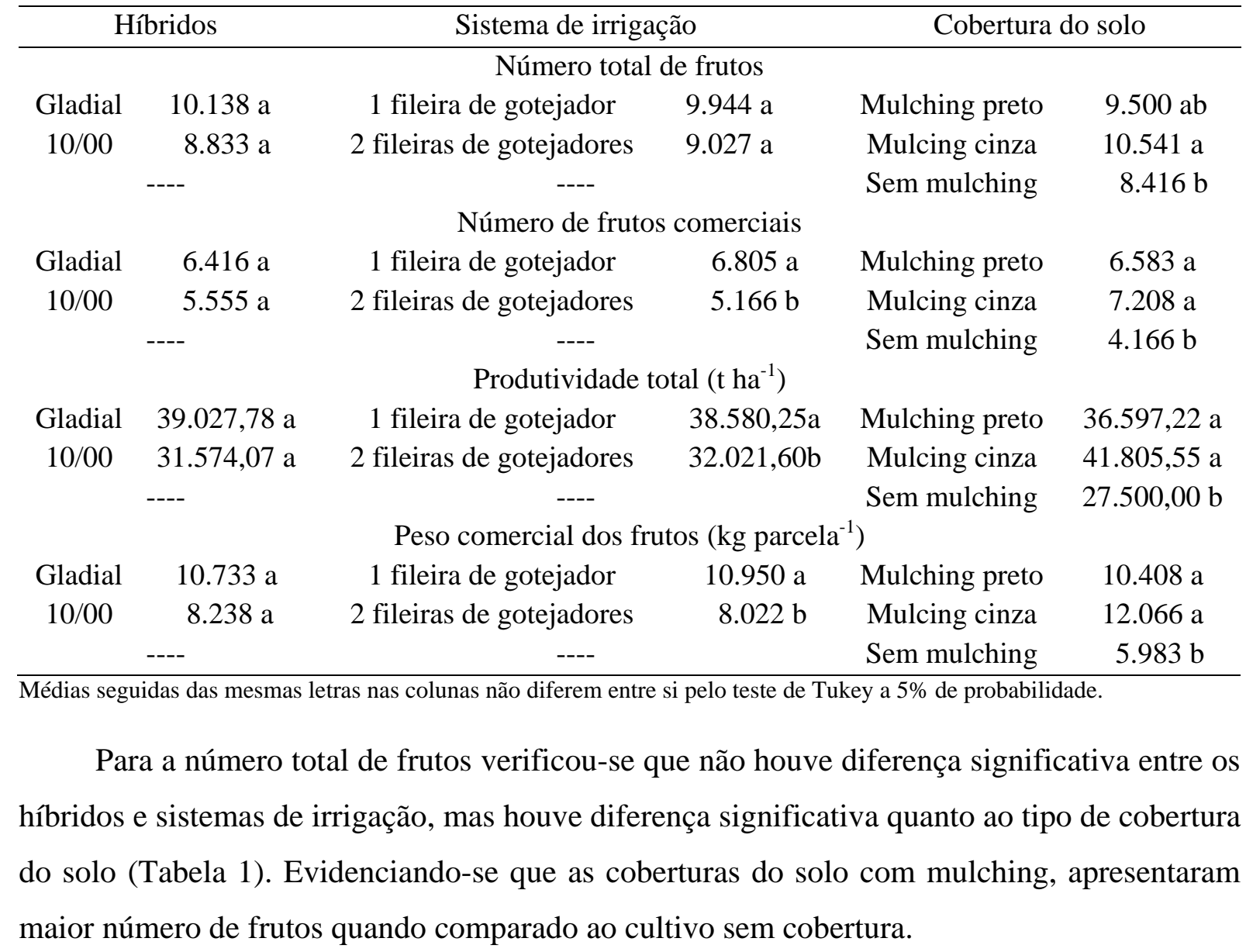


Foram observados que para o número comercial de frutos não houve diferença significativa entre os híbridos, mas houve diferença significativa quanto ao sistema de irrigação e o tipo de cobertura do solo (Tabela 1). Evidenciando-se que as coberturas do solo com mulching, apresentaram maior número de frutos quando comparado ao cultivo sem cobertura, pode-se ser associado ao não contato direto do fruto com o solo.

Já quanto a produtividade total e peso comercial dos frutos constatou-se que não houve diferença significativa entre os híbridos, mas houve diferença significativa quanto ao sistema de irrigação e o tipo de cobertura do solo (Tabela 1). A produtividade total alcançada pelo experimento foi superior à média nacional que é de 26,82 t ha ${ }^{-1}$ (IBGE, 2015). Sousa et al (2010), conseguiram produtividade máxima de $34,8 \mathrm{t} \mathrm{ha}^{-1}$, examinando diferentes lâminas de irrigação para as cultivares Goldmine e 10-00.

Os melhores índices produtivos e qualitativos no trabalho foram conseguidos significativamente associados ao uso de uma linha de gotejamento e integrados com uma cobertura do solo através de mulching, com produtividade máxima de 41.805,55 t ha ${ }^{-1}$ (Tabela 1). Isso corrobora com trabalho realizado por Medeiros et al. (2006), que verificou que cultivos de melão manejados com mulching apresentou produtividades de até $39 \%$ superior em comparação aos cultivos sem mulching. Entretanto em trabalho realizado com as cultivares de melões Goldmine e 10-00, submetidos à diferentes lâminas de irrigação por gotejamento, com e sem filmes plásticos no solo, obtiveram produtividade máxima de $38,1 \mathrm{t} \mathrm{ha}^{-1}$, em solo sem filmes plásticos (Simões et al., 2016).

A cor do mulching utilizados também influenciou significativamente o desempenho produtivo do meloeiro, observando que os melhores resultados em uma comparação númerica foram percebidos primeiramente com o mulching cinza e em seguida o mulching preto. De acordo com o trabalho feito por Gondim et al. (2009), constataram que a cobertura do solo com plásticos de cor marrom, apresentaram melhores respostas de qualidades dos melões.

Isso pode ser explicado pelo fato da quantidade de energia absorvida e refletida depende no uso do mulching (Varejão-Silva, 2006), e isso mantém uma área molhada mais prolongada devido a diminuição da temperatura do solo e consequentemente evaporação de água, disponibilizando assim maior oferta hídrica da matriz do solo para a planta.

Quanto a diferença significativa para os dois sistemas de irrigação, pode estar associada, com a porcentagem de solo molhado, o sistema com uma linha de gotejamento apresentou menor porcentagem de solo molhado mais uma maior disponibilidade de água em profundidade, sendo uma situação oposta para o sistema com duas linhas de gotejamento. 
Na figura 4, observa-se o aumento da tensão de água no solo registrada ao longo das fases fenológicas da cultura, evento ocorrindo possivemente pela maior demanda hídrida por parte da cultura, devido à diminuição da lâmina advindo do coeficiente de cultura (kc) baixo.

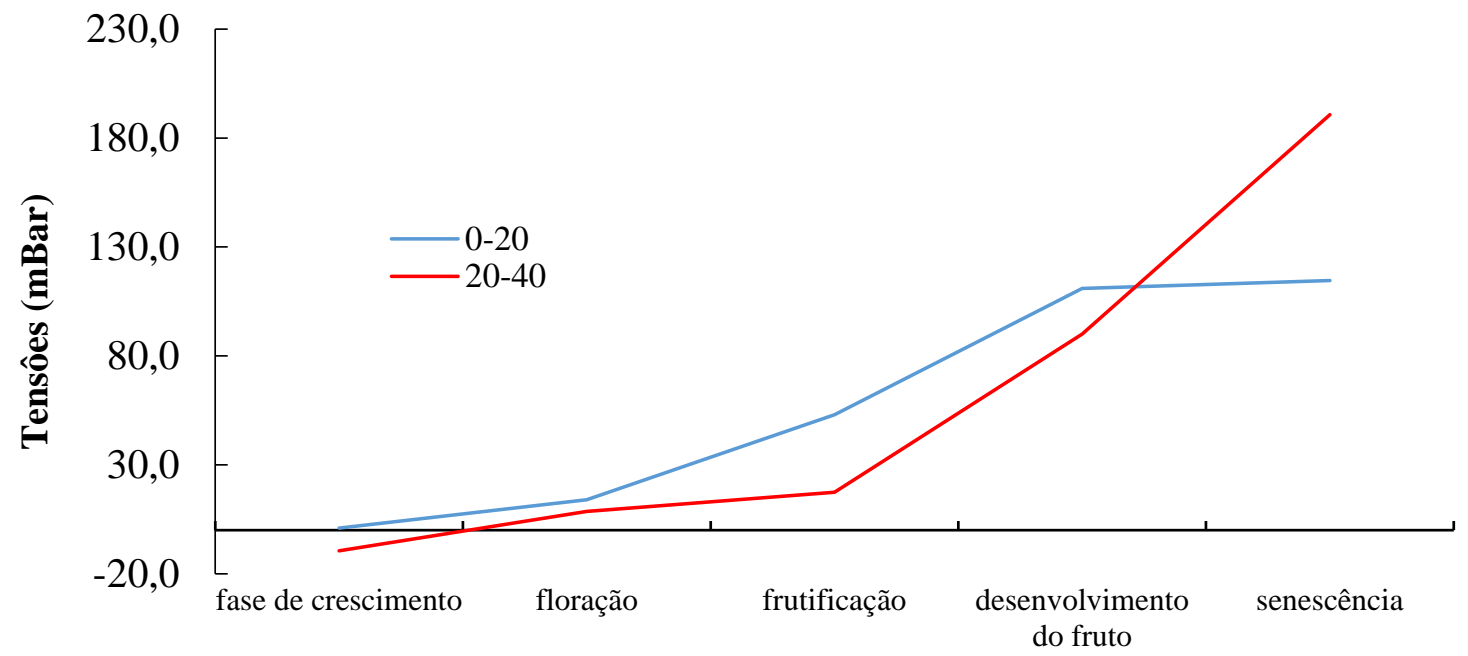

Figura 4. tensões observadas nas profundidades de 0-20 e 20-40 cm ao longo das diferentes fases fenológicas da cultura do meloeiro.

$\mathrm{Na}$ tabela 2, estão registrados as tensões durante o ciclo completo do melão, com maior tensão de água no solo registrada ao longo das fases fenológicas da cultura.

Tabela 2. Valores de tensão registrados para os híbridos de melão amarelo submetidos a diferentes sistemas de irrigação e coberturas do solo. Petrolina, IFSERTÃO-PE, 2016.

\begin{tabular}{|c|c|c|c|c|c|}
\hline \multirow{3}{*}{$\begin{array}{c}\text { Profundidade }(\mathrm{cm}) \\
0-20\end{array}$} & \multicolumn{5}{|c|}{ Tensões nas fases fenológicas (mBar) } \\
\hline & fase de & floraçã & frutificaçã & desenvolvimento do & senescênci \\
\hline & crescimento & o & o & fruto & a \\
\hline 1 fileira de gotejador & 0,82 & 14,97 & 82,13 & 144,38 & 187,70 \\
\hline $\begin{array}{l}2 \text { fileiras de } \\
\text { gotejadores }\end{array}$ & 1,21 & 12,93 & 23,87 & 77,61 & 41,53 \\
\hline $20-40$ & $\begin{array}{c}\text { fase de } \\
\text { crescimento }\end{array}$ & $\begin{array}{c}\text { floraçã } \\
\text { o }\end{array}$ & $\begin{array}{c}\text { frutificaçã } \\
0\end{array}$ & $\begin{array}{l}\text { desenvolvimento do } \\
\text { fruto }\end{array}$ & $\begin{array}{c}\text { senescênci } \\
\mathrm{a}\end{array}$ \\
\hline 1 fileira de gotejador & $-7,93$ & 2,87 & 27,21 & 159,68 & 307,10 \\
\hline $\begin{array}{l}2 \text { fileiras de } \\
\text { gotejadores }\end{array}$ & $-11,03$ & 14,41 & 7,66 & 20,39 & 74,42 \\
\hline
\end{tabular}

A maior intensidade nas tensões no sistema de irrigaçao com 1 linha de gotejador, pode ser possivemente reflexo de uma menor área de bulbo molhado no solo, em relação ao sistema com duas linhas de gotejamento. 


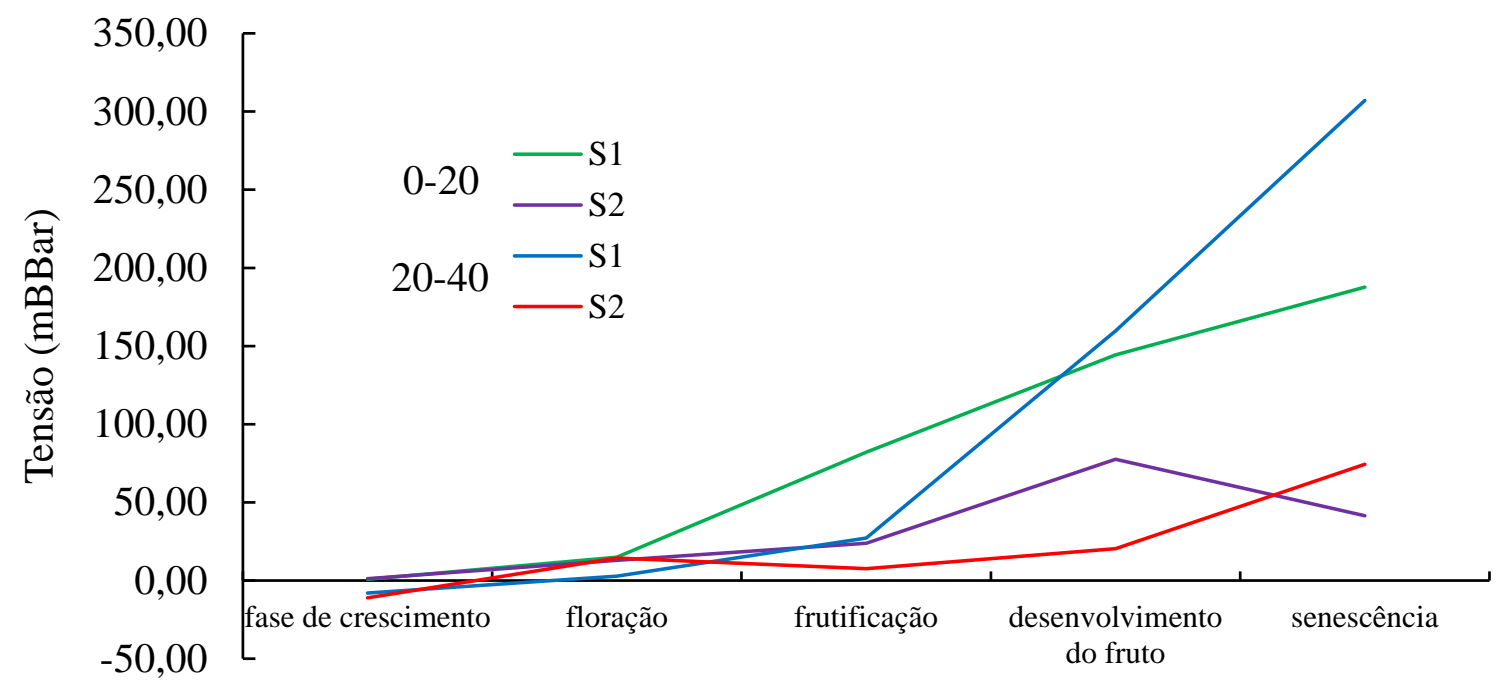

Figura 5. gráfico com tensões observadas nos dois sistemas de irrigação ao longo das diferentes fases fenológicas da cultura do meloeiro nas profundidades de $0-20$ e $20-40 \mathrm{~cm}$.

\section{CONCLUSÃO}

Houve efeito significativo apenas para produtividade dos melões Gladial e 10/00 quanto à cobertura de solo.

A maior produtividade encontrada foi de 41.805,55 $\mathrm{t} \mathrm{ha}^{-1}$ para o melão Gladial e o 10/00 com sistema de irrigação por gotejamento com 1 fileira de gotejador por planta e cobertura de solo com mulching cinza.

\section{REFERÊNCIAS}

ABREU, T. A.; OLITTA, A. F. L.; MARCHETTI, D. A. Comparação de métodos de irrigação por sulco e por gotejo na cultura do melão, no Vale do São Francisco. Pesquisa Agropecuária Brasileira, Brasília, v.13, n.3, 1978. p 35-45.

ALLEN, R. G.; PEREIRA, L. S.; RAES, D.; SMITH, M. Crop evapotranspiration: guidelines for computing crop water requirements. Rome: FAO, FAO. Irrigation and Drainage Paper, 56. 1998. 297p.

AZEVEDO, P.V.; SILVA, B.B.; SILVA, V.P.R. Walter requirements of irrigated mango orchards in Northeast Brazil. Agricultural Water Management 58: 2003. p 241-245. 
BATISTA, P. F.; PIRES, M. M. M. L.; SANTOS, J. S.; QUEIROZ, S. O. P.; ARAGÃO, C. A.; DANTAS, B. F. Produção e qualidade de frutos de melão submetidos a dois sistemas de irrigação. Horticultura Brasileira, 27. 2009. p 246-250.

BERNARDO, S.; SOARES, A. A.; MANTOVANI, E. C. Manual de irrigação. 8.ed. Viçosa: Ed. UFV, 2006. 625p.

CAVALCANTI, F. J. A., coord. Recomendações de adubação para o Estado de Pernambuco: $2^{a}$ aproximação. 3.ed. Recife, IPA, 2008. 212p.

CRISÓSTOMO, L. A. et al. Adubação, irrigação, híbridos e práticas para o meloeiro no Nordeste. Fortaleza: EMBRAPA, (Circular Técnica, 14). 2002. 22p.

EMBRAPA, Empresa Brasileira de Pesquisa Agropecuária - Semiárido. Dados meteorológicos automáticos. Disponível em: <emprapasepatsa_dadosmeteorlogicosbebedouro.com.br> acessado em 20 abr. 2016.

GOMES, H. P. Engenharia de irrigação: hidráulica dos sistemas pressurizados, aspersão e gotejamento. 3.ed. Campina Grande: Ed. UFPB, 1999. 412 p.

GONDIM, et al. Qualidade de melão 'Torreon' cultivado com diferentes coberturas de solo e lâminas de irrigação. Revista Ceres, vol. 56, núm. 3, mayo-junio, Universidade Federal de Viçosa, Vicosa, Brasil. 2009. p. 326-331.

IBGE. Anuário Estatístico do Brasil, v. 75, 2015. Produção agrícola municipal 2013-2014. In: IBGE. Sidra: sistema IBGE de recuperação automática. Rio de Janeiro, [2015]. tab. 1612.Disponívelem:<http://servicodados.ibge.gov.br/Download/Download.ashx?http=1\&u=bi blioteca.ibge.gov.br/visualizacao/periodicos/20/aeb_2015.pdf>. Acesso em: jan. 2016.

LEMOS, L.M.C.; SANTOS, S.R.; LEMOS, J.P. Crescimento do meloeiro Cantaloupe sob manejo de irrigação por gotejamento em Janaúba, Minas Gerais. Rev. Ceres, Viçosa, v. 59, n.5, 2012. p. 614-623.

LIMA, E.M.C. Irrigação do meloeiro cultivado em ambiente protegido. Lavras: UFLA. Tese de doutorado em Recursos hídrico em sistemas agrícolas. Universidade Federal de Lavras. 2015. 139p.

MANTOVANI, E. C.; BERNARDO, S.; PALARETTI, L. F. Irrigação: princípios e métodos. 3 ed., atualizada e ampliada, Viçosa: Ed. UFV, 2009. 355p. 
W. de B. Pereira et al.

SHMUELI, M. \& GOLDBERG, D. Riego por aspersión, por surcos y por Goteo dei Meián en una Zona Anda. Israel, Universidade Hebrea de Jerusalem, 1971. 5 p.

SILVA, M. C. C.; MEDEIROS, J. F.; NEGREIROS, M. Z.; SOUSA, V. F. Produtividade de frutos do meloeiros sob diferentes níveis de salinidade da água de irrigação, com e sem cobertura do solo. Horticultura Brasileira, Brasília, v.23, n.2, 2005. p. 202-205.

SILVA, FAS Assistat assistência estatística. Versão 7.7 beta. 2014. Disponível em: <http://www.assistat.com/indexi.html>. Acesso em: 7 mar. 2017.

SIMÕES, W. L. et al. Uso de filmes plásticos no solo para o cultivo de meloeiro irrigado. Water Resources and Irrigation Management. Universidade Federal do Recôncavo da Bahia, Cruz das Almas, BA. Instituto Federal Baiano, Salvador, BA v.5, n.1, Jan.-Abr. 2016. p 23-29.

SOUSA, A. E. C. et al. Produtividade do meloeiro sob lâmina de irrigação e adubação potássica. Eng. Agríc. vol.30, n.2, 2010. p. 271-278.

VERMEIREN, G. A., JOBLING, G. A. Irrigação localizada. Campina Grande: UFPB, (Estudos FAO: Irrigação e Drenagem, 36 - Tradução de GHEYI, H. R., DAMASCENO, F. A. V., SILVA JUNIOR, L. G. A., MEDEIROS, J. F.). 1997. 184p.

VAREJÃO-SILVA, M. A. Meteorologia e Climatologia. Versão Digital 2. Recife, Pernambuco, 2006. 463p. 